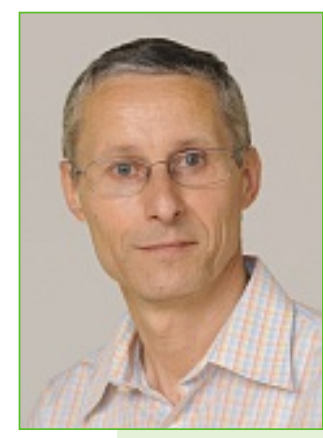

\title{
Wie gefährlich sind eigentlich Phytotherapeutika?
}

Umfang und Häufigkeit v.a. populärwissenschaftlicher Darstellungen von unerwünschten Wirkungen (UAW) pflanzlicher Arzneimittel in den Medien erwecken bisweilen den Eindruck, Phytotherapeutika seien nicht nur von fraglichem therapeutischen Nutzen, sondern auch hinsichtlich ihrer Nebenwirkungen im Vergleich zu synthetischen Arzneimitteln besonders problematisch und es sei daher dringend erforderlich, vor ihnen zu warnen. Tatsächlich zeichnen sich aber gerade die traditionell gebräuchlichen europäischen Arzneipflanzen bei sachgerechter Anwendung durch besonders gute Verträglichkeit aus.

Basis der heutigen Phytotherapie ist das ursprüngliche Wissen über Heilpflanzen aus der traditionellen Volksheilkunde unter Berücksichtigung der modernen naturwissenschaftlich fundierten Erkenntnisse über die pharmakologischen Wirkungen von Naturstoffen für rationale Arzneimittelentwicklungen. Das Wirkstoffgemisch aus der Arzneipflanze führt im Organismus zu einer komplexen Gesamtwirkung, deren einzelne Wirkkomponenten sich nur mittels großen Aufwands darstellen lassen. Deshalb beschränkt man sich in Zulassungsverfahren für den Wirkungsnachweis häufig auf sicher erfassbare Detailwirkungen. Hieraus resultiert zwangsläufig eine Unterschätzung des therapeutischen Potenzials und dies hat mitunter Einfluss auf die Gewichtung von Haupt- und Nebenwirkungen.

Wie eine Reihe von Beispielen aus der jüngeren Vergangenheit zeigt, führen tatsächliche oder vermeintliche toxische Nebenwirkungen bei Arzneipflanzen sehr rasch zum Anwendungsverbot entsprechender Zubereitungen, gegenüber Nebenwirkungen von synthetischen Pharmaka existiert offenbar eine größere Toleranz. Allerdings habe ich den Eindruck, dass hier mit zweierlei Maß gemessen wird, denn während für Kava-Kava-Extrakte die Inzidenz an hepatotoxischen Nebenwirkungen pro 1 Million Tagesdosen mit 0,008 Fällen angegeben wurde, beträgt sie für Diazepam 2,12 Fälle!
Warum kommt es immer wieder zu einer Diskussion der Toxizität von Phytotherapeutika? Dafür gibt es mehrere Ursachen:

$>$ unkritische Übertragung von Vergiftungsfällen bei Tieren auf Menschen

$>$ kritikloses Aufgreifen von Meldungen zu unerwünschten Wirkungen

$>$ unkritische Verallgemeinerungen

$>$ theoretisch formulierte Risiken ohne experimentelle Beweise oder klinische Befunde

> Überbewertung von Einzelfällen

> Missbrauch der Pharmakovigilanz

Selbstverständlich kann mit zunehmendem therapeutischem Einsatz die Zahl unerwünschter Ereignisse steigen. Den möglichen ursächlichen Zusammenhang dieser unerwünschten Ereignisse mit der Verabreichung des Phytotherapeutikums gilt es in jedem Fall kritisch zu prüfen und bei Notwendigkeit auch Konsequenzen zu ziehen.

Ein tendenziöser Medizinjournalismus und selbsternannte Experten erwecken zudem den Eindruck, es gäbe keine modernen Studien zur Wirkung und Sicherheit von Arzneipflanzen und rationalen Phytotherapeutika, die wissenschaftlichen Kriterien standhalten. Stattdessen werden längst widerlegte Anekdoten erzählt.

Und was lehrt uns dies? Forschung und Aufklärung sind nach meinem Erachten die einzigen Mittel, um eine ungerechtfertigte Debatte zur Toxizität von Arzneipflanzenzubereitungen zu verhindern bzw. ihr wissenschaftlich fundiert entgegenzutreten.

In der Verantwortung steht auch diese Zeitschrift, die durch ihre Beiträge die Diskussion versachlichen, Vorurteile entkräften und falsche Behauptungen widerlegen helfen sollte. Dazu mögen sich viele Kolleginnen und Kollegen angesprochen fühlen!

Ihr

Matthias F. Melzig 